\title{
Minimally Invasive Extraforaminal Lumbar Interbody Fusion for Revision Surgery: A Technique through Kambin's Triangle
}

\author{
Jun Gue Lee ${ }^{1}$, Hyeun Sung Kim², Seok Won Kim ${ }^{1}$ \\ ${ }^{1}$ Department of Neurosurgery, Chosun University College of Medicine, Gwangju, \\ ${ }^{2}$ Department of Neurosurgery, Nanoori Hospital, Suwon, Korea
}

\begin{abstract}
Objective: The purpose of this study was to evaluate the clinical outcomes of minimally invasive extraforaminal lumbar interbody fusion (ELIF) for revision surgery.

Methods: From January 2011 to December 2012, 12 patients who underwent minimally invasive ELIF through the Kambin's triangle for revision surgery were included in this study. All patients underwent the surgical procedure in the following sequence: (1) epidural anesthesia, (2) exposing the Kambin's triangle toward the lateral part of the dura (partial resection of the superior articular process), (3) bilateral cage insertion for reinforcement of stabilization and fusion, and (4) percutaneous transpedicular screwing. Clinical outcomes were assessed using the visual analogue scale (VAS), and Oswestry disability index (ODI). Imaging and clinical findings including surgical techniques, clinical outcomes, and related complications were depicted and analyzed.

Results: The mean age of the patients ( 5 men, 7 women) was $60.7 \pm 13.4$ years, and the mean follow-up period was $27.1 \pm 4.9$ months. The mean VAS (back and leg) score improved significantly at final follow-up. The mean ODI score decreased as follows: preoperative, $76.78 \pm 6.08$; 3 months after the surgery, $37.74 \pm 6.67$; and at final follow-up, $29.91 \pm 2.98$. Two patients presented with transient nerve root irritation, but there were no cases of incidental dural tear or serious infection. No significant neurological deterioration or major complication was noted in any of the patients.
\end{abstract}

Conclusion: Minimally invasive ELIF for revision surgery is an effective surgical option with a low complication rate.

Key Words: Spine $\cdot$ Fusion $\cdot$ Reoperation

\section{INTRODUCTION}

Epidural fibrosis encountered during revision surgery alters the anatomic landmarks and natural planes, which can result in accidental durotomy or neural injury, ${ }^{8,9}$. Besides degenerative spondylolisthesis, the major indications for lumbar interbody fusion include revision surgery for recurrent disc herniation or post-laminectomy instability. Lumbar interbody fusion techniques have been developed and modified over the years to improve fusion rates and clinical outcomes in the treatment of degenerative lumbar spine disease.

- Received: July 7, 2015 • Revised: November 9, 2015

- Accepted: November 16, 2015

Corresponding Author: Seok Won Kim, MD, PhD

Department of Neurosurgery, Chosun University College of Medicine,

365 Pilmun-daero, Dong-gu, Gwangju 61453, Korea

Tel: +82-62-220-3126, Fax: +82-62-227-4575

E-mail:ns64902@hanmail.net/chosunns@chosun.ac.kr

$\otimes$ This is an Open Access article distributed under the terms of the Creative Commons Attribution Non-Commercial License (http://creativecommons.org/ licenses/by-nc/3.0/) which permits unrestricted non-commercial use, distribution, and reproduction in any medium, provided the original work is properly cited.
Both posterior lumbar interbody fusion (PLIF) and transforaminal lumbar interbody fusion (TLIF) are now standard procedures in the spinal surgeons' armamentarium for treatment of degenerative lumbar spine diseases ${ }^{5}$. Moreover, in comparison to an open procedure, minimally invasive surgery (MIS) with the goal of reducing iatrogenic soft tissue injury is now becoming the standard technique for spine surgery. MIS is associated with lesser blood loss, lower risk of transfusion, shorter hospital stay, and comparable short-term outcomes without an increased risk of significant perioperative morbidity ${ }^{4,7}$. See comment in PubMed Commons below.

Extraforaminal lumbar interbody fusion (ELIF) is performed by accessing the intervertebral disc between the exiting nerve root and the superior articular process of the lower vertebral segment; this site is referred to as the Kambin's triangle ${ }^{1)}$.

Theoretically, for revision surgery, availability of a site different from the old scar tissue for gaining access is a great advantage. Until now, however, there has been no study that has specifically addressed applicability of this newly emerging ELIF technique for revision surgery. In this study, we aimed to assess the clinical results of the minimally invasive revision ELIF with a longer than 2-year follow-up in 12 patients. 


\section{MATERIALS AND METHODS}

Between January 2011 and November 2012, 12 consecutive patients underwent minimally invasive ELIF for revision surgery. The mean age of the patients was $60.7 \pm 14.6$ years $(5$

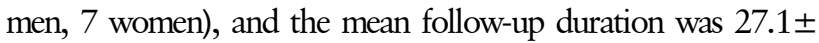
3.2 months. The inclusion criteria were as follows: (1) recurrent disc herniation, recurrent stenosis, or post-laminectomy instability, with a pain-free interval of at least 6 months' duration at the same level, (2) primarily radicular symptoms with an acute onset, and (3) correlating neurodiagnostic symptoms and correlating positive magnetic resonance imaging findings.

All patients were required to receive conservative treatment for at least 3 months without relief before being considered for minimally invasive ELIF. The patients' preoperative demographics are shown in Table 1.

\section{Surgical Technique}

The procedure was performed under epidural anesthesia with patient in the prone position and with hips and knees slightly flexed to maintain lumbar lordosis. Posterior skin incision was made approximately $5 \mathrm{~cm}$ long and $6-10 \mathrm{~cm}$ lateral from the midline. Following this, the intertransverse space was accessed through a cleavage between the medial multifidus and lateral longissimus, and they were separated via blunt digital dissection. The base of the superior articular process and the neural foramen were palpated from outside the spinal canal to confirm the existence of the exiting nerve root.
In this approach, the base of the superior articular process is the first structure to be removed (Fig. 1). As the inferior articular process overlies the dorsal side of superior articular process, this approach can preserve inferior articular process. Unlike TLIF technique, complete facetectomy is usually not necessary to allow visualization and removal of the intervertebral disc.

Surgical removal of the base of the superior articular process is initiated and continued in an arcuate manner and a supero-medial direction to expose the lateral border of the ligamentum flavum covering the exiting nerve root and the infero-medial limits of the pedicle. The fibrocartilage and the lateral border of the ligamentum flavum were removed with Kerrison punches to expose the exiting nerve root. Further dissection revealed the dorsal root ganglion (DRG) (Fig. 2). Any soft tissue attachments including extraforaminal ligaments were separated to release the exiting nerve root and the DRG to facilitate free mobilization of the nerve root. The room for exposing and preparing the disc is limited to the Kambin's triangle (Fig. 3).

Following exposure, the root was gently retracted and microscopic discectomy was performed without any obstacles. The endplate was prepared using chisel for bone grafting. A polyetheretherketone cage packed with allograft bone chips was inserted under C-arm fluoroscopic guidance. This procedure was then repeated on the contralateral side. After interbody fusion, percutaneous screw fixation was performed (Fig. 4).

\section{Safety and Outcome Evaluation}

The visual analogue scale (VAS) pain score and Oswestry disability index (ODI) were used to assess the intensity of back

Table 1. Clinical characteristics of patients who underwent revision ELIF

\begin{tabular}{|c|c|c|c|c|c|c|}
\hline Case & Age/Sex & Level & First surgery & $\begin{array}{c}\text { Symptom-free duration (months) } \\
\text { after first surgery }\end{array}$ & Complications & $\begin{array}{c}\text { Follow-up duration } \\
\text { (months) }\end{array}$ \\
\hline 1 & $67 / F$ & L3-4 & Laminectomy & 12 & - & 26 \\
\hline 2 & $74 / M$ & L4-5 & Laminectomy & 24 & - & 26 \\
\hline 3 & $57 / F$ & L4-5 & Discectomy & 16 & Transient radiculopathy & 29 \\
\hline 4 & $48 / F$ & L4-5 & Laminectomy & 10 & - & 26 \\
\hline 5 & $62 / M$ & $\llcorner 4-5$ & Discectomy & 8 & - & 25 \\
\hline 6 & $60 / F$ & L4-5 & Discectomy & 7 & - & 32 \\
\hline 7 & $65 / M$ & L4-5 & Laminectomy & 14 & - & 28 \\
\hline 8 & $70 / F$ & L4-5 & Laminectomy & 15 & Transient radiculopathy & 24 \\
\hline 9 & $71 / M$ & L4-5 & Laminectomy & 24 & - & 25 \\
\hline 10 & $58 / F$ & L3-4 & Laminectomy & 13 & - & 30 \\
\hline 11 & $47 / F$ & $\llcorner 4-5$ & Discectomy & 22 & Superficial infection & 26 \\
\hline 12 & $49 / M$ & L4-5 & Laminectomy & 20 & - & 28 \\
\hline
\end{tabular}




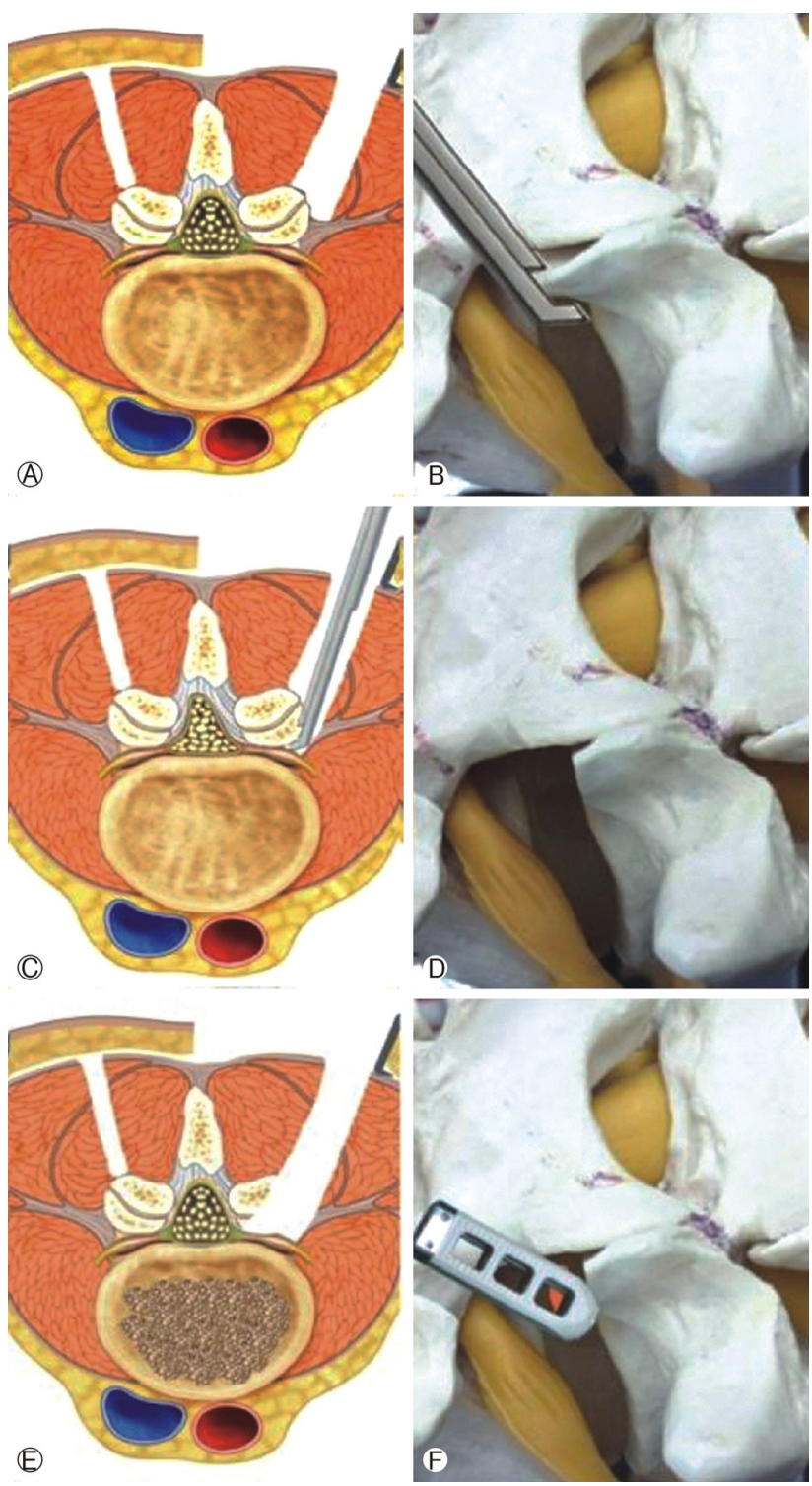

Fig. 1. Diagram of surgical technique. (A, B) The multifidus and longissimus muscles are separated. (C, D) Removal of superior articular process. (E, F) Discectomy and cage insertion.

and leg pain and the functional outcome at different time points. The patients were evaluated according to a modified version of MacNab criteria for characterizing the clinical outcomes after spine surgery.

\section{Statistical Analysis}

Statistical analysis was performed using SAS 6.12 (SAS Institute Inc., Cary, NC, USA). Mean values and standard deviations were calculated. Parameters were compared among time points using the Wilcoxon signed-rank test. p-value of 0.05 was considered indicative of statistical significance.

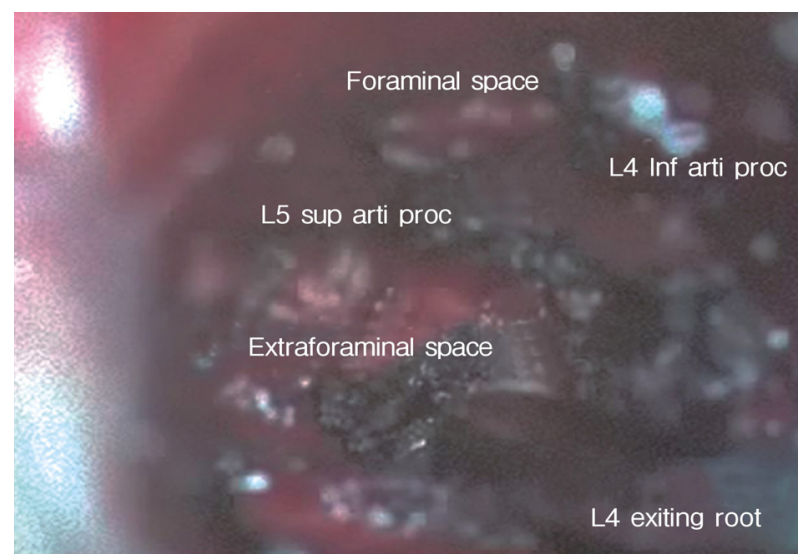

Fig. 2. Surgical details of right $L 4-5$ intertransverse area for extraforaminal lumbar interbody fusion.

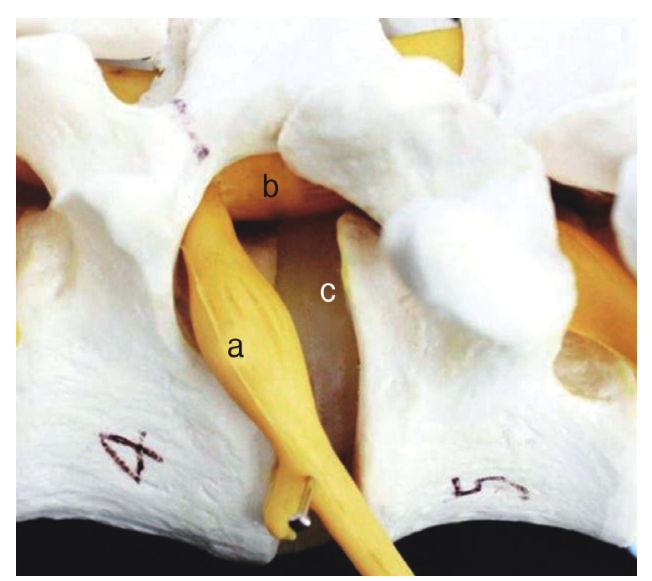

Fig. 3. Schematic representation of Kambin's safety triangle (laterally by the exiting nerve root (a), medially by the lateral margin of the traversing nerve (b), caudally by the upper endplate of the lower vertebra (c)).

\section{RESULTS}

The ELIF procedure was performed under epidural anesthesia, intravenous analgesia with opioid medication, and 4-10 mg midazolam sedation. The fused levels represented in this study were L4-5 $(n=10)$ and L3-4 $(n=2)$.

\section{Clinical Outcomes}

Excellent or good results were achieved in all patients based on the modified MacNab's criteria.

The mean ODI score was $76.78 \pm 6.08$, which improved to $29.91 \pm 2.98$ at the final follow-up $(p<0.001)$. The mean VAS (back pain) score also improved from $6.43 \pm 1.04$ preopera- 
tively to $3.04 \pm 0.71$ at the final follow-up $(p<0.001)$. The mean VAS (leg pain) score was $7.70 \pm 0.70$ preoperatively and $2.91 \pm 0.73$ at the final follow-up $(\mathrm{p}<0.001)$ (Table 2).

\section{Radiological Outcomes}

At the final follow-up, none of the 12 patients appeared to have fusion failure as defined by the presence of a definite radiolucent line around the pedicle screws or cages and more than $5^{\circ}$ of motion on dynamic flexion and extension views. Any signs of implant breakdown or loosening were investigated.

\section{Complications}

Complications occurred in three patients. One patient had a superficial wound infection, which resolved with intravenous and oral antibiotics. Two patients experienced sciatica and sensory changes during the early postoperative period, and these were completely resolved within a month. There
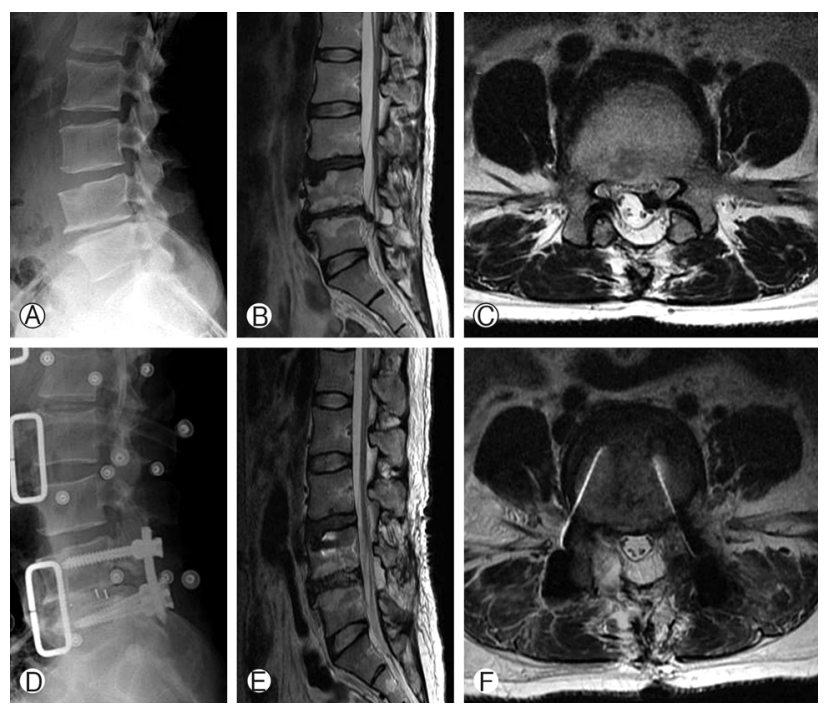

Fig. 4. A 74 year-old man who underwent decompressive laminectomy 2 years ago. (A, B, C) Preoperative simple radiograph and T2-weighted magnetic resonance images show left-sided recurrent disc herniation at the L4-5 level. (D, E, F) Simple radiograph and T2-weighted magnetic resonance images taken 3 months after ELIF reveal complete removal of the ruptured disc material and appropriate cage insertion.

Table 2. Mean (standard deviation) clinical outcome scores

\begin{tabular}{lrrc}
\hline \hline Outcome & Pre-operative & Final follow-up & $\begin{array}{c}\text { p-value (Wilcoxon } \\
\text { signed-rank test) }\end{array}$ \\
\hline ODI & $76.78 \pm 6.08$ & $29.91 \pm 2.98$ & $<0.001$ \\
VAS-BP & $6.43 \pm 1.04$ & $3.04 \pm 0.71$ & $<0.001$ \\
VAS-LP & $7.70 \pm 0.70$ & $2.91 \pm 0.71$ & $<0.001$ \\
\hline
\end{tabular}

was no dural tear in association with epidural fibrosis, which can occur frequently in revision surgery. There were no permanent neurological sequelae due to the minimally invasive ELIF.

\section{DISCUSSION}

Revision surgeries are defined as secondary operations in patients who have previously undergone traditional discectomy or decompressive laminectomy at the same level. Revision surgery is generally considered to be more technically demanding and stressful than primary surgery and may be associated with higher complication rates, especially those of neural injuries and incidental dural tears ${ }^{2,14)}$.

Traditionally, open posterior fusion techniques have been used commonly for revision surgery. However, these techniques require extensive tissue dissection to gain access to the disc space and provide the ideal lateral-to-medial orientation for optimal screw trajectory; additionally, these can result in denervation and atrophy of muscles. During the clinical followup, significant postoperative muscle atrophy, loss of function, and increased pain have been evidenced ${ }^{3,11)}$.

Moreover, revision spine surgery is challenging and has been reported to pose a greater risk for complications due to the distorted anatomy and epidural fibrosis. Both PLIF and TLIF techniques are now widely accepted and performed for revision surgery; however, in these circumstances, extensive dissection of the paravertebral musculature and fibrotic scar increases the risk of neural injury and incidental dural tears ${ }^{8}$.

The one of the most common complications of conventional revision lumbar surgery is an incidental dural tear, and it varies from 8 to $21 \% 0^{9,13)}$.

Theoretically, it is obvious that the lateral transforaminal approach for ELIF bypasses the dorsal part of the scar tissue and diminishes the risk of an unintended dural tear ${ }^{10)}$. However, to the best of the authors' knowledge, no previous study has evaluated the effects of ELIF as revision surgery after microscopic discectomy or decompressive laminectomy.

Selznick et al. ${ }^{13)}$ reported that as a revision surgery, even minimally invasive TLIF was associated with more frequent dural tears compared with primary surgery (29\% versus $4 \%$ ) and had a higher perioperative complication rate.

ELIF has a more lateral access approach than TLIF, avoiding the inferior articular process and allowing for full exposure of the superior counterpart, which causes myelopathy or radiculopathy. It also facilitates the indirect decompression of the lateral vertebral canal and the fusion of vertebral bodies in the "Kambin's safety triangle".

The disc is removable without interference from the scar tissue. In comparison, dorsal reintervention requires scar removal, tedious mobilization, and retraction of the compressed 
nerve root. A PLIF procedure, especially altered for revision surgery, presents with the risks of direct lesions of the dural sac and the cauda equine with hematoma or postoperative fibrosis. Some reports have depicted foraminal approaches, which are unlike the classical posterior approach. In the Wiltse paraspinal approach, the lumbar spine is accessed through the similar cleavage path between the multifidus and longissimus muscles, as in the ELIF approach. However, there are some distinctions between the two to approach the intervertebral disc. The Wiltse's technique starts with a skin incision near the midline; the approach is relatively sagittal and has some risks of back muscle injury. On the contrary, the ELIF procedure, uses more lateral trajectory. It makes exposing the lateral part of the disc and exiting nerve root easy without disturbing abdominal cavity, posterior midline structures and inferior articular process. Resection of superior articular process only can expose the intervertebral foramen that is located distal to the dura. To avoid previous scar tissue, an anterior approach can be preformed for revision surgery ${ }^{12}$. The anterior approach presents with risks inherent in retroperitoneal surgery, such as digestive and more importantly, vascular and neurological risks. On the other hand, by respecting the vascular and digestive structures, the more laterally oriented ELIF technique avoids the risks of severe bleeding and potentially, a more dangerous dissection of the spinal canal ${ }^{6,12)}$.

In this study, we demonstrate the favorable outcomes of ELIF for revision surgery with a minimum 2-year follow-up. There was no occurrence of a dural tear, and it had a $90 \%$ excellent or good success rate. We therefore regard revision ELIF as a good option for minimally invasive interbody fusion.

However, the ELIF technique may have some limitations. Severe instability or high grade spondylolisthesis are inadequate indication for ELIF. In case of the L5-S1 level that is situated on very deep between the iliac crests, it is not easy to approach due to its steep angle. The more lateral approach of ELIF makes the access much deeper than TLIF, so it is greater burden to light operation field and perform surgery, especially in obese patients. These limitations do not allow satisfactory access path to the intervertebral disc and safe procedure at the L5-S1 level. One more drawback of this technique includes its disposition of indirect decompression. Despite our successful results, it is hard to achieve excellent outcome for a subset of patients due to failure of indirect decompression. In particular, it is difficult to decompress traversing root in recurrent disc herniation. For this reason, spinal instability, foraminal disc and thickened yellow ligament at the foramen can be better surgical indications for minimally invasive ELIF.

Novice practitioners should therefore gather ripe experience in minimally invasive ELIF before attempting it in the revision spine surgery.

\section{CONCLUSION}

Our results show that minimally invasive revision ELIF at the L3-4 and L4-5 levels is an effective surgical option for reducing complications with no violation of the posterior tension structures and anterior vascular structures. It has the added advantage of avoiding the previous surgical scar.

\section{REFERENCES}

1. Baek OK, Lee SH: Extraforaminal lumbar interbody fusion for the treatment of isthmic spondylolisthesis. J Spinal Disord Tech 22:219-227, 2009

2. Cammisa FP Jr, Girardi FP, Sangani PK, Parvataneni HK, Cadag S, Sandhu HS: Incidental durotomy in spine surgery. Spine (Phila Pa 1976) 25:2663-2667, 2000

3. Fan S, Hu Z, Zhao F, Zhao X, Huang Y, Fang X: Multifidus muscle changes and clinical effects of one-level posterior lumbar interbody fusion: minimally invasive procedure versus conventional open approach. Eur Spine J 19:316-324, 2010

4. Foley KT, Holly LT, Schwender JD: Minimally invasive lumbar fusion. Spine (Phila Pa 1976) 28:26-35, 2003

5. Humphreys SC, Hodges SD, Patwardhan AG, Eck JC, Murphy $\mathrm{RB}$, Covington LA: Comparison of posterior and transforaminal approaches to lumbar interbody fusion. Spine (Phila Pa 1976) 26:567-571, 2001

6. Ikard RW: Methods and complications of anterior exposure of the thoracic and lumbar spine. Arch Surg 141:1025-1034, 2006

7. Isaacs RE, Podichetty VK, Santiago P, Sandhu FA, Spears J, Kelly K, et al: Minimally invasive microendoscopy-assisted transforaminal lumbar interbody fusion with instrumentation. J Neurosurg Spine 3:98-105, 2005

8. Jun BY: Posterior lumbar interbody fusion (PLIF) by facetolaminoplasty in lumbar spine revision surgery. J Korean Neurosurg Soc 28:1746-1754, 1999

9. Khan IS, Sonig A, Thakur JD, Bollam P, Nanda A: Perioperative complications in patients undergoing open transforaminal lumbar interbody fusion as a revision surgery. J Neurosurg Spine 18:260-264, 201

10. Kim MY, Kim SW, Kim SH, Kim HS: Extraforaminal lumbar interbody fusion for cage migration after screw removal: A case report. Korean J Spine 10:104-106, 2013

11. Liu X, Wang Y, Wu X, Zheng Y, Jia L, Li J, et al: Impact of surgical approaches on the lumbar multifidus muscle: an experimental study using sheep as models. J Neurosurg Spine 12: 570-576, 2010

12. Recoules-Arche D, Druschel C, Fayada P, Vinikoff L, Disch AC: Unilateral Extra-Foraminal Lumbar Interbody Fusion (ELIF): Surgical Technique and Clinical Outcome in 107 Patients. J Spinal Disord Tech [Epub ahead of print], 2014

13. Selznick LA, Shamji MF, Isaacs RE: Minimally invasive interbody fusion for revision lumbar surgery: technical feasibility and safety. J Spinal Disord Tech 22:207-213, 2009

14. Wang JC, Bohlman HH, Riew KD: Dural tears secondary to operations on the lumbar spine. Management and results after a two-year-minimum follow-up of eighty-eight patients. J Bone Joint Surg Am 80:1728-1732, 1998 